\title{
Estudos biomecânicos da ação de aderências sobre anastomose cólica. Trabalho experimental em ratos $^{1}$
}

\author{
Feng Chung $\mathrm{Wu}^{2}$ \\ Maria de Lourdes Setsuko Ayrizono ${ }^{2}$ \\ João José Fagundes ${ }^{3}$ \\ Cláudio Sadi Rodrigues $\mathrm{Coy}^{3}$ \\ Juvenal Ricardo Navarro Góes ${ }^{4}$ \\ Luis Sérgio Leonardi ${ }^{5}$
}

\begin{abstract}
Wu FC, Ayrizono MLS, Fagundes JJ, Coy CSR, Góes JRN, Leonardi LS. Estudos biomecânicos da ação de aderências sobre anastomose cólica: trabalho experimental em ratos. Acta Cir Bras [serial online] 2003 Maio-Jun;18(3). Disponível em URL: http://www.scielo.br/acb.
\end{abstract}

RESUMO - Objetivo: Avaliar a ação de aderências sobre anastomose em cólon descendente de ratos por meio de análises biomecânicas. Métodos: Noventa ratos foram distribuídos igualmente em três grupos. O Grupo 1 submeteu-se ao teste Pressão de Ruptura à Distensão por Líquido, enquanto que os Grupos 2 e 3, aos testes Força de Ruptura à Tração e Tensão Resultante Final de Ruptura à Tração, respectivamente. Cada grupo foi subdividido em 3 subgrupos contendo 10 animais. Um subgrupo foi analisado com as aderências deixadas intactas ao redor da anastomose; em outro, as aderências foram removidas, enquanto no último não se permitiu a fixação de aderências nas anastomoses por meio de proteção com película de polivinilcloreto (P.V.C.). Resultados: A eutanásia ocorreu no quinto dia pós-operatório e os subgrupos com as aderências intactas nas anastomoses apresentaram maior resistência à ruptura em relação aos subgrupos com aderências removidas. Os subgrupos com ausência de aderências nas anastomoses apresentaram menor resistência à ruptura (Grupo1-p=0,0001, Grupo 2-p=0,0003 e Grupo3-p=0,0001). Conclusão: Aderências constituem influências benéficas sobre a anastomose, aumentando a sua resistência mecânica na fase inicial do reparo tecidual.

DESCRITORES - Cicatrização. Cólon. Aderências. Anastomose. Biomecânica.

\section{Introdução}

Os fundamentos da cicatrização cólica têm sido amplamente estudados em trabalhos clínicos e experimentais, pois deiscências em anastomoses nessa região representam importante causa de morbidade e mortalidade ${ }^{(1,2,3,4,5,6)}$. Verifica-se na prática cirúrgica um fenômeno que ocorre conjuntamente à evolução do

1. Trabalho realizado no Núcleo de Cirurgia Experimental - Disciplina de Moléstias do Aparelho Digestivo do Departamento de Cirurgia - Serviço de Colo-Proctologia da Faculdade de Ciências Médicas da Universidade Estadual de Campinas - UNICAMP.

2. Pós-graduando em Cirurgia - Serviço de Colo-Proctologia da Faculdade de Ciências Médicas da Universidade Estadual de Campinas - UNICAMP.

3. Professor Doutor da Disciplina de Moléstias do Aparelho Digestivo do Departamento de Cirurgia - Serviço de Colo-Proctologia da Faculdade de Ciências Médicas da Universidade Estadual de Campinas - UNICAMP.

4. Professor Associado da Disciplina de Moléstias do Aparelho Digestivo do Departamento de Cirurgia - Chefe do Serviço de ColoProctologia da Faculdade de Ciências Médicas da Universidade Estadual de Campinas - UNICAMP.

5. Professor Titular e Chefe da Disciplina de Moléstias do Aparelho Digestivo do Departamento de Cirurgia da Faculdade de Ciências Médicas da Universidade Estadual de Campinas - UNICAMP. 
processo de cicatrização das anastomoses intestinais, a formação de aderências. Essas estruturas podem levar à variações na reparação tecidual da linha de sutura apresentando desde efeitos deletérios como obstrução intestinal $^{(7)}$ e influências benéficas, como ações protetoras biológicas e mecânicas ${ }^{(4,5)}$.

Este estudo foi proposto com o objetivo de se investigar a ação de aderências sobre anastomoses no cólon descendente de rato em um período crítico da cicatrização, o quinto dia pós-operatório. Para esse fim, foram aplicados os testes biomecânicos uniaxiais Pressão de Ruptura à Distensão por Líquido ${ }^{(8,9)}$ (PRDL) e Força de Ruptura à Tração ${ }^{(10)}($ FRT), além da introdução de um método biomecânico biaxial, a Tensão Resultante Final de Ruptura à Tração (TRF) $)^{(11)}$.

\section{Métodos}

Este trabalho foi aprovado pela Comissão de Ética na Experimentação Animal CEEA-IB-UNICAMP, segundo os Princípios Éticos adotados pelo Colégio Brasileiro de Experimentação Animal (COBEA) - Protocolo $n^{\circ}$ 327-1. Foram utilizados 90 ratos machos, Wistar-CEMIB/UNICAMP, com massa corporal variando de 270 a 320 gramas e criados sob condições ambientais e alimentares semelhantes. A umidade e a temperatura eram as mesmas do ambiente geral e o ciclo claro/escuro de 12 horas cada. A ração foi suspensa nas 24 horas que precediam as cirurgias, mantendose livre o suprimento de água. Os 90 ratos foram distribuídos em 3 grupos e cada grupo subdividido em 3 subgrupos contendo 10 animais (Tabela 1).

TABELA 1 - Distribuição dos grupos e subgrupos com as suas características.

\begin{tabular}{c|c|l}
\hline Grupo & Subgrupo & \multicolumn{1}{|c}{ Característica do segmento de alça durante os testes } \\
\hline \multirow{2}{*}{1} & 1.1 & Aderências deixadas intactas ao redor da anastomose \\
\cline { 2 - 3 }$($ PRDL) & 1.2 & Aderências removidas da anastomose \\
\cline { 2 - 3 } & 1.3 & Ausência da formação de aderências na anastomose (proteção por película - P.V.C) \\
\hline \multirow{2}{*}{2} & 2.1 & Aderências deixadas intactas ao redor da anastomose \\
\cline { 2 - 3 }$($ FRT) & 2.2 & Aderências removidas da anastomose \\
\cline { 2 - 3 } & 2.3 & Ausência da formação de aderências na anastomose (proteção por película - P.V.C) \\
\hline \multirow{2}{*}{3} & 3.1 & Aderências deixadas intactas ao redor da anastomose \\
\cline { 2 - 3 }$($ TRF) & 3.2 & Aderência removida da anastomose \\
\cline { 2 - 3 } & 3.3 & Ausência da formação de aderências na anastomose (proteção por película - P.V.C) \\
\hline
\end{tabular}

O teste PRDL foi aplicado no Grupo 1, enquanto que nos Grupos 2 e 3, os testes FRT e TRF, respectivamente. A anestesia foi endovenosa (veia caudal) com pentobarbital sódico à $3 \%$ diluído em solução fisiológica (5 mg/ml) em dose de $30 \mathrm{mg} / \mathrm{kg}$ e o local de anastomose situou-se no cólon descendente a $2,5 \mathrm{~cm}$ da reflexão peritoneal. Após secção transversal do cólon, a anastomose foi realizada com fio de polipropileno 8-0, término-terminal, com pontos seromusculares, separados, extra-mucosos com justaposição das bordas. Nos animais pertencentes aos subgrupos 1.3, 2.3 e 3.3, cobriu-se a área da anastomose com uma película de P.V.C. para impedir a fixação de aderências. A colocação da película foi feita com fio de polipropileno 7-0 em mesocólon e músculo íleolombar esquerdo. A eutanásia ocorreu no quinto dia pós-operatório com dose letal de tiopental sódico à $3 \%$ (veia caudal). A cavidade abdominal era aberta e um segmento de cólon de cinco centímetros com a anastomose no seu ponto médio era retirado e submetido aos procedimentos de acordo com o subgrupo pertencente (Figura 1). Antes dos testes, os espécimes foram mergulhados em solução contendo cloridrato de papaverina e solução fisiológica $(250 \mathrm{mg} /$ litro $)$ à $37^{\circ} \mathrm{C}$, por um período de 30 minutos. 

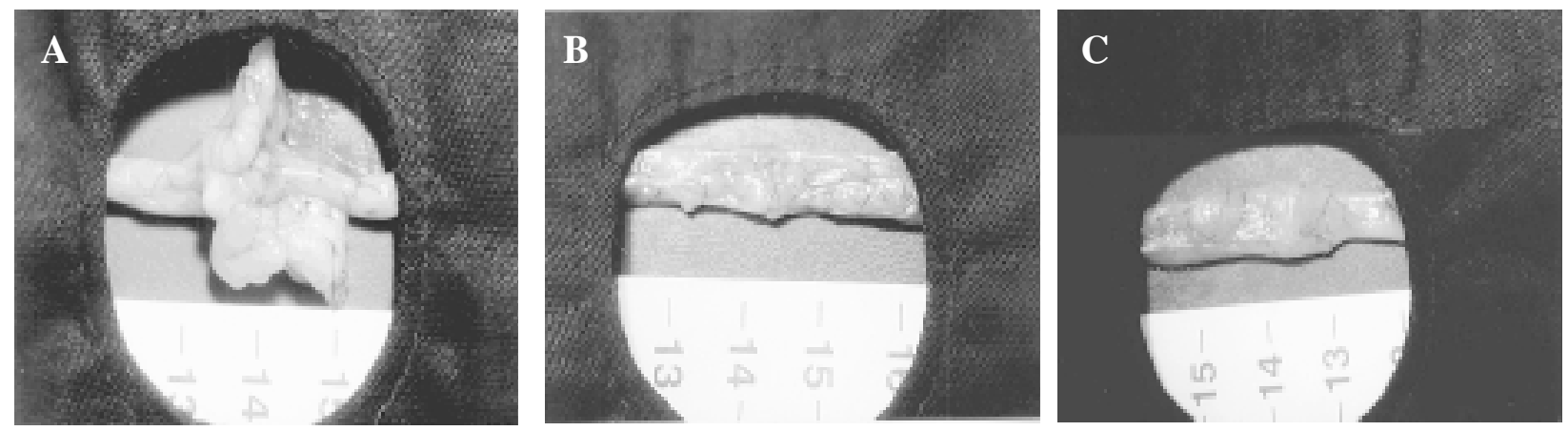

FIGURA 1 - (A) Segmento de cólon com as aderências intactas. (B) Segmento de cólon com as aderências removidas. (C) Segmento de cólon com ausência de aderências (proteção P.V.C.).

\section{Pressão de Ruptura à Distensão por Líquido - PRDL (Grupo 1)}

O teste PRDL foi definido como a pressão intraluminar, em milímetros de mercúrio, necessária para promover o extravasamento da solução infundida, através do ponto de ruptura da anastomose. O espécime foi acoplado a torneira de tripla-via (NIPRO $\rightarrow$-Luerlock) já instalada na seringa da bomba de infusão (B.BRAUN-871.012) e no sistema de sustentação. Um cateter de polietileno conectou o sistema ao polígrafo (MINGOGRAF-804/SIEMENS-ELEMA) e instilou-se solução fisiológica preenchendo-o, formando assim um conjunto de vasos comunicantes (Figura 2).

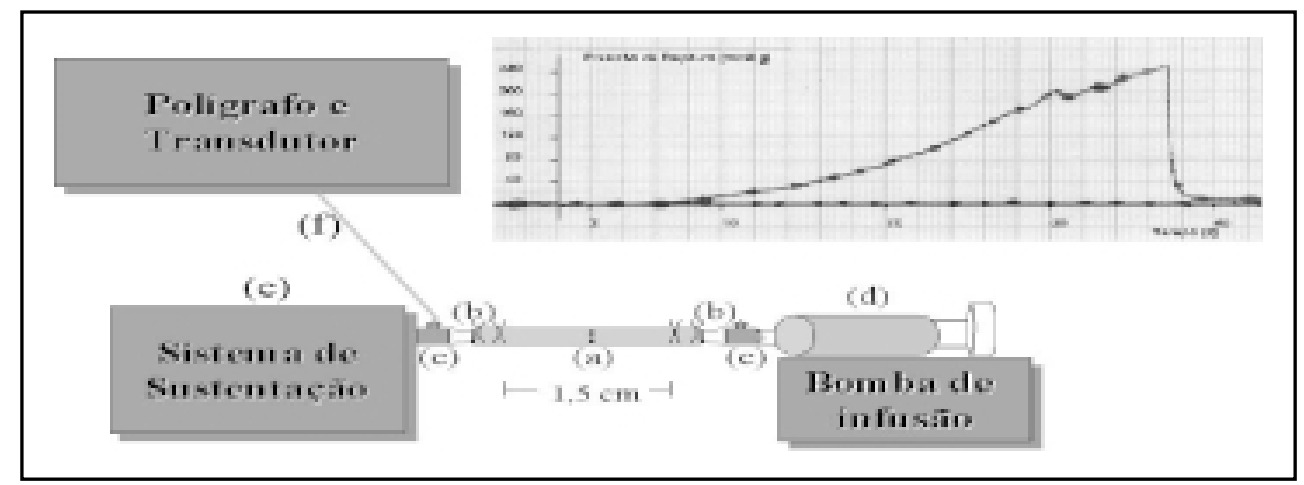

FIGURA 2 - Representação esquemática do teste Pressão de Ruptura à Distensão por Líquido e o seu respectivo registro gráfico (Pressão x Tempo): a) segmento de cólon, (b) canhões do cateter de ${ }^{\circ} 18$, (c) torneiras de tripla-via, (d) seringa de vidro usada para infusão, (e) sistema de sustentação e (f) cateter de polietileno. (Seta) Momento de ruptura da anastomose.

Tomou-se essa situação como parâmetro inicial zero e o estudo da PRDL foi iniciado pela infusão de solução fisiológica em velocidade constante de $2 \mathrm{ml} /$ minuto e cessado após a queda no registro da pressão.

\section{Força de Ruptura à Tração - FRT (Grupo 2)}

O teste FRT foi definido como a força de tração, em gramas-força, necessária para romper a anastomose. $\mathrm{O}$ espécime foi posicionado no sistema de tração e um cateter de polietileno (PE-80) conectouo ao polígrafo (MINGOGRAF-804/SIEMENSELEMA). Instilou-se então solução fisiológica para o interior do sistema e tomou-se essa situação como inicial zero. A tração ocorreu na velocidade de $1 \mathrm{~cm} / \mathrm{min}$ e a força foi determinada por meio de um dinamômetro (Käfer-RenéGraf). Com a tração, originou-se uma pressão no sistema que foi registrada pelo polígrafo e na ruptura, a curva retornou à linha de base. Uma filmadora (CCD-TRV65-DigitalZoom 220X) captou o número marcado pelo dinamômetro sendo esse valor inserido na seguinte equação:

$$
\mathrm{FRT}=\mathrm{X} .21,351+96
$$

Esta equação originou-se a partir da aferição do dinamômetro.

( $\mathbf{X}$ = valor de leitura do dinamômetro no instante de ruptura da anastomose)(Figura 3). 

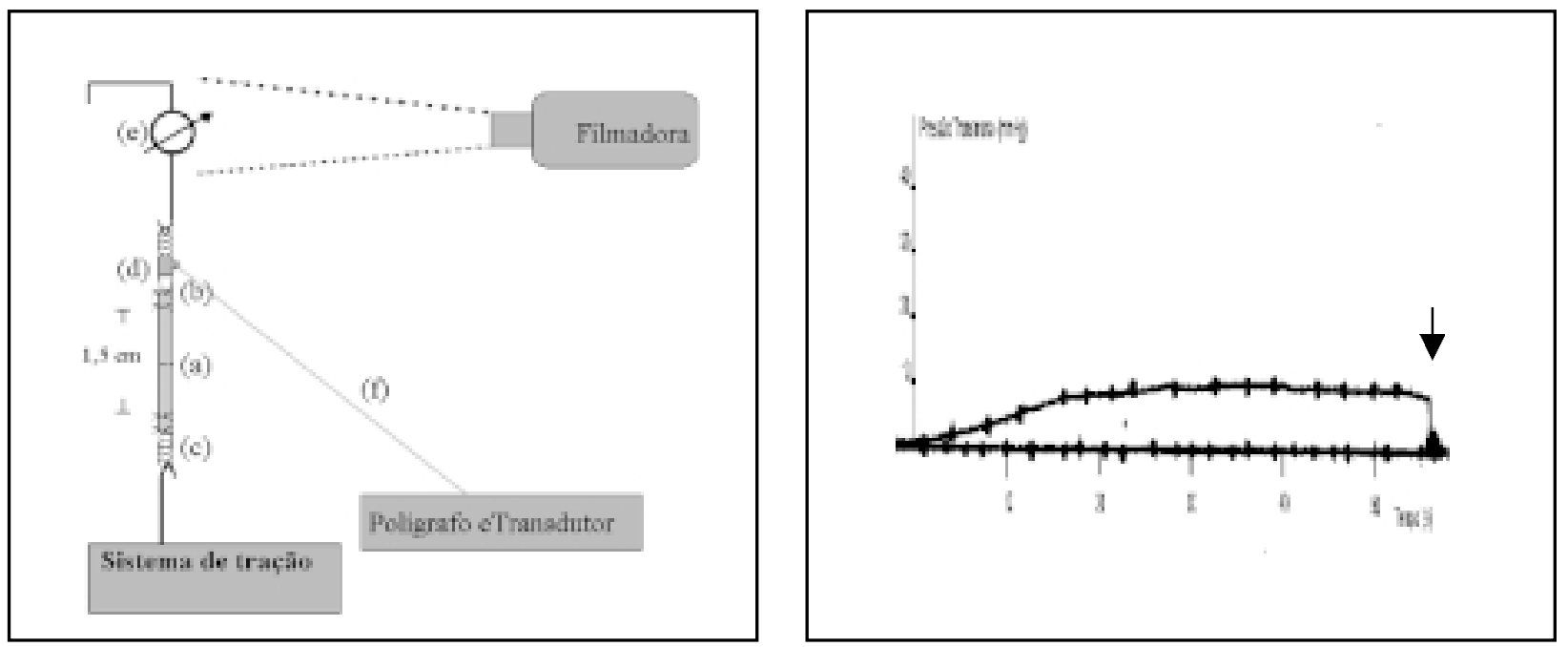

FIGURA 3 - Representação esquemática Força de Ruptura à Tração e o gráfico da Pressão transmural (Pressão x Tempo): (a) segmento de cólon, (b) canhão do cateter $n^{\circ} 18$-luz aberta, (c) canhão do cateter $n^{\circ} 18$-luz vedada e fixo na corrente, (d) torneira de tripla-via, (e) dinamômetro com a filmadora posicionada no seu marcador e (f) cateter de polietileno. (Seta) Momento de ruptura.

\section{Tensão Resultante Final de Ruptura à Tração - TRF (Grupo 3)}

Para a realização do estudo da Tensão Resultante Final de Ruptura à Tração (TRF) foram necessários os cálculos da tensão resultante longitudinal (TRL) e da tensão circunferencial (TC) da alça cólica no momento de ruptura, ambas expressas em dina/ $\mathrm{cm}^{2}$. O sistema de tração utilizado para a obtenção do TRF foi similar ao método FRT, com o acréscimo de dois componentes: uma seringa de insulina para medir o volume de líquido no interior da alça e uma outra filmadora (modelo CCDTRV65-DigitalZoom 220X) para captar a imagem do estiramento da alça até o momento do rompimento. Neste instante, dois vetores ortogonais agem sobre o ponto de ruptura: a tensão circunferencial e a tensão resultante longitudinal. A composição de ambas é a
Tensão Resultante Final de Ruptura à Tração (TRF) e pode ser calculada pela seguinte fórmula (Teorema de Pitágoras)(Figura 4): $\quad \mathbf{T R F}^{2}=\mathbf{T R L}^{2}+\mathbf{T C}^{\mathbf{2}}$

$\cap$ A Tla foi calculada segundo a equação ${ }^{(12)}$ :

$\mathbf{T l a}=\mathbf{F R T} /\left(\pi \cdot \mathbf{r}_{\mathrm{e}}{ }^{2}-\pi \cdot \mathbf{r}_{\mathrm{i}}{ }^{2}\right)$

$\cap$ FRT $=$ Força de Ruptura à Tração;

$r_{e}=$ raio externo; $r_{i}=$ raio interno

$\cap$ A Tl foi calculada segundo a equação ${ }^{(12)}$ :

$$
\mathbf{T}_{1}=\left(\mathbf{P t} \cdot \mathbf{r}_{\mathrm{i}}\right) / 2 \text {. } \mathrm{e}
$$

$\cap$ A TC foi calculada segundo a equação ${ }^{(12)}$ :

$\mathbf{T}_{\mathrm{C}}=\left(\mathbf{P t} \cdot \mathbf{r}_{\mathrm{i}}\right) / \mathbf{e}$

$\cap \mathrm{Pt}=$ Pressão transmural;

$\mathrm{r}_{\mathrm{i}}=$ raio interno; $\mathrm{e}=$ espessura da parede no instante de ruptura

$\cap$ A TRL é dada pela somatória da TL + Tla

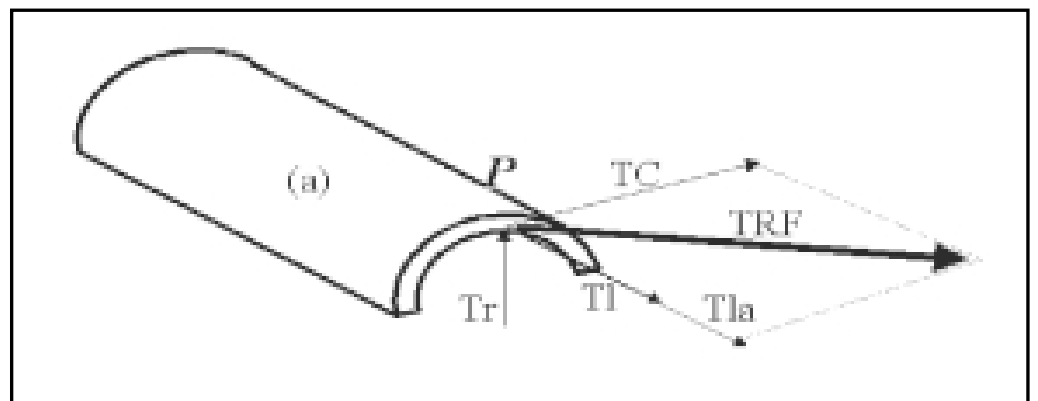

FIGUKA 4 - Kepresentaçao esquematıca das tensoes exercıaas no ponto de ruptura da alça (P): (TRF) tensão resultante final de ruptura à tração, (TC) tensão circunferencial, (Tl) tensão longitudinal, (Tla) tensão longitudinal adicional, (Tr) tensão radial, $(\mathrm{Tl}+\mathrm{Tla})$ tensão resultante longitudinal e (a) alça secionado longitudinalmente. 
Volume da alça (v), Força de ruptura à Tração (FRT) e Pressão transmural (Pt)

Com o sistema montado (Figura 5) e preenchido com solução fisiológica, realizou-se um balanço no polígrafo. Por meio de sucção pela seringa de insulina pré-posicionada no polígrafo, aspirou-se o líquido do sistema para o seu interior. Esse volume representa o volume do segmento de alça, pois, quando o líquido deixa o seu lúmen, ocorre a formação de vácuo interrompendo a aspiração. Introduzindo novamente a solução da seringa para o sistema, o lúmen da alça era preenchido e o registro do polígrafo que apresentavase negativo pela aspiração, retorna à linha de base inicial. Esse volume foi confirmado como o volume real do segmento de alça. A FRT e a Pt foram obtidos pelo método descrito anteriormente (Figura 3).

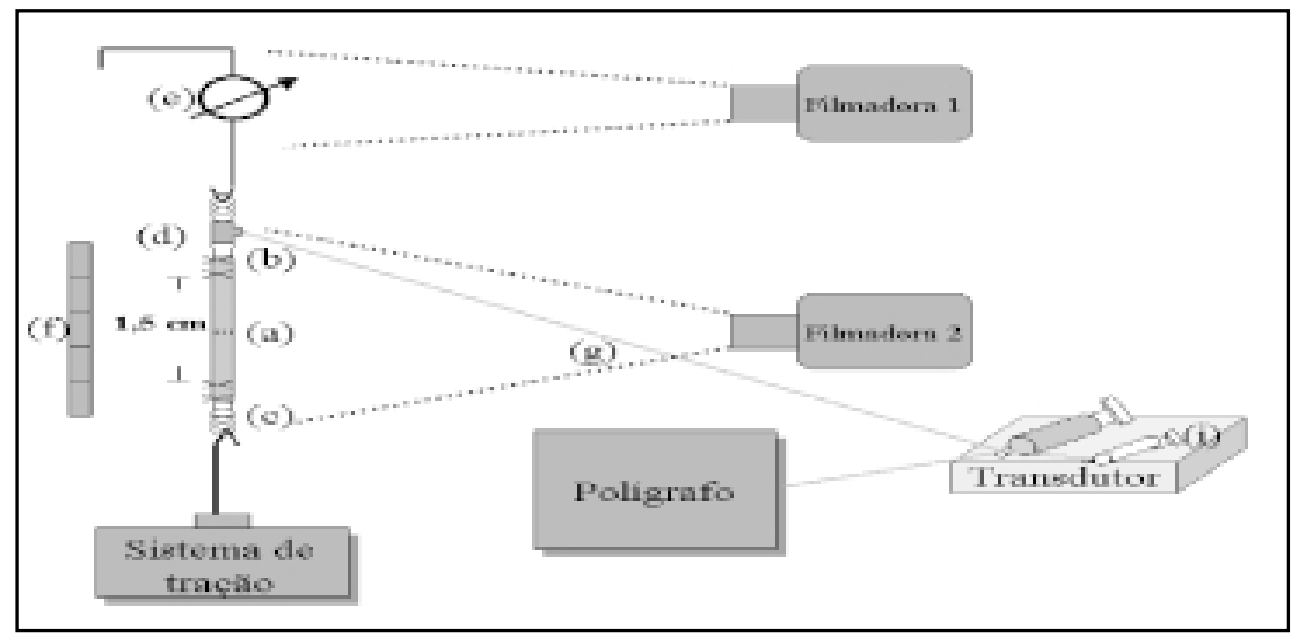

FIGURA 5 - Representação esquemática do teste Tensão Resultante Final de Ruptura à Tração: (a) segmento de cólon (filmadora 2), (b) canhão de cateter $\mathrm{n}^{\circ} 18$ - luz aberta, (c) canhão de cateter $\mathrm{n}^{\circ}$ 18-luz vedada fixo à corrente, (d) torneira de tripla-via fixa à corrente, (e) dinamômetro (filmadora 1), (f) régua fixada em mesmo plano da alça, (g) cateter de polietileno e (i) seringa de insulina vazia.

Comprimento da alça cólica no momento de ruptura $(h)$

O cálculo do $\mathbf{h}$ foi dado pela equação:

$h=1,5+T t .1 / 60$ - dl

$1,5=$ comprimento inicial; $\mathrm{Tt}=$ tempo total $\mathrm{de}$ tração; $1 / 60$ = velocidade de tração;

$\mathrm{dl}=$ deslocamento da alça de arrasto do dinamômetro

Raio interno $\left(r_{i}\right)$, Raio externo $\left(r_{e}\right)$ e espessura da parede da alça no instante de ruptura (e)

$\mathrm{O} \mathbf{r}_{\mathrm{i}}$ da alça na ruptura foi calculado por meio da equação ${ }^{(12)}: \quad \quad \mathbf{r}_{i}{ }^{2}=\mathbf{v} /(\pi \cdot \mathbf{h})$

$\mathrm{V}=$ volume final $\mathrm{h}=$ comprimento final da alça.

A equação representa o cálculo do raio interno de um cilindro, pois a alça assume essa morfologia sob tração. $\mathrm{O}$ volume inicial é igual ao final (v) pois não ocorre perda de líquido intraluminar até o momento de ruptura. Os testes eram então registrados por uma filmadora e as imagens transferidas para a estação de trabalho O2 da Silicon Graphics-Media Tools. Essas imagens foram repassadas quadro a quadro e congelado no momento exato anterior à ruptura. A alça configurouse na tela do computador como um retângulo e por meio de "zoom", os lados menores (diâmetro externo da alça) foram medidas pelo sistema de coordenadas. $\mathrm{O} \mathbf{r}_{\mathrm{e}}$ corresponde a metade do diâmetro externo e a diferença entre o $\mathbf{r}_{\mathrm{e}}$ com o $\mathbf{r}_{\mathrm{i}}$ será a espessura (e).

As análises estatísticas utilizadas para as avaliações dos resultados foram os testes não-paramétrico de Kruskal-Wallis e de Mann-Whitney. Em todos os testes foi fixado o nível de rejeição da hipótese de nulidade o valor $\mathrm{p}<0,05$.

\section{Resultados}

Os animais apresentaram boa evolução clínica e as rupturas ocorreram sempre nas anastomoses. Os resultados dos testes PRDL, FRT e TRF estão distribuídos conforme a tabela 2. 
TABELA 2 - Distribuição dos valores da média, mínimo, máximo e desvio-padrão dos subgrupos pertencentes aos Grupo 1 (mmHg), 2 (grama-força) e $3\left(\mathrm{dina} / \mathrm{cm}^{2}\right)$.

\begin{tabular}{c|c|c|c|c|c}
\hline Subgrupos & $\mathbf{N}$ & Média & Mínimo & Máximo & Desvio-padrão \\
\hline 1.1 & 10 & 213,8 & 200 & 244 & 14,0618 \\
\hline 1.2 & 10 & 183,4 & 144 & 216 & 24,4413 \\
\hline 1.3 & 10 & 146,0 & 120 & 176 & 18,7853 \\
\hline 2.1 & 10 & 202,883 & 151,726 & 267,449 & 40,5460 \\
\hline 2.2 & 9 & 143,304 & 105,608 & 210,228 & 44,5476 \\
\hline 2.3 & 9 & 113,496 & 100,270 & 141,264 & 14,8665 \\
\hline 3.1 & 10 & 3170281,46 & 2037638,93 & 5274751,40 & 1172086,35 \\
\hline 3.2 & 10 & 1892149,83 & 944470,23 & 2721093,52 & 617117,08 \\
\hline 3.3 & 10 & 1073048,45 & 594508,97 & 2006381,78 & 529210,82 \\
\hline
\end{tabular}

Teste Pressão de Ruptura à Distensão por Líquido - PRDL (Grupo 1)

Aplicando o método estatístico Kruskal-Wallis, os subgrupos 1.1, 1.2 e 1.3 apresentaram diferenças significante para $\mathrm{p}=0,0001$. No teste Mann-Whitney, o subgrupo 1.1 em relação ao $1.2,1.1$ em relação ao 1.3 e 1.2 em relação ao 1.3 também foram diferentes entre si para $\mathrm{p}=0.0085, \mathrm{p}=0,0001$ e $\mathrm{p}=0,003$, respectivamente.

\section{Teste Força de Ruptura à Tração - FRT (Grupo 2)}

Dois animais foram excluídos desse grupo, um deles em função de dois pontos de sutura serem removidas junto com as aderências e outro pela presença de aderências na linha de sutura. Os subgrupos 2.1, 2.2 e 2.3 apresentaram diferença significante $(\mathrm{p}=0,0003)$ (Kruskal-Wallis). No teste Mann-Whitney, o subgrupo 2.1 em relação ao 2.2, 2.1 em relação ao 2.3 e 2.2 em relação ao 2.3 apresentaram também diferenças significantes $(\mathrm{p}=0.0127, \mathrm{p}=0,0002 \mathrm{e}$ $\mathrm{p}=0,0377)$, respectivamente.

\section{Teste Tensão Resultante Final de Ruptura à Tração - TRF (Grupo 3)}

Aplicando o método Kruskal-Wallis, os subgrupos 3.1, 3.2 e 3.3 apresentaram diferenças significantes $(\mathrm{p}=0,0001)$. No teste Mann-Whitney, o subgrupo 3.1 em relação ao $3.2,3.1$ em relação ao 3.3 e $3.2 \mathrm{em}$ relação ao 3.3 também foram diferentes entre si $(\mathrm{p}=0,0091, \mathrm{p}=0,0002$ e $\mathrm{p}=0,0113)$, respectivamente.

\section{Discussão}

Aderências inevitavelmente formam-se após intervenções cirúrgicas abdominais e representam a maior causa de obstrução intestinal mecânica ${ }^{(7,13)}$. Todavia, a maioria das aderências são inofensivas e estão envolvidas em ações protetoras nas anastomoses atuando basicamente em duas áreas: biológica e mecânica. A primeira consiste na formação de neovascularização, na melhora do processo de drenagem linfática e na capacitação fagocitária, enquanto que a segunda se caracteriza pelo efeito selador nas falhas da anastomose, comportando-se como "cola fisiológica" ${ }^{\prime 2,4,5,7)}$.

Neste estudo, a ação de aderências no quinto dia pós-operatório foi avaliada por meio de três testes biomecânicos, a Pressão de Ruptura à Distensão por Líquido (PRDL) ${ }^{(9,6,14)}$ e a Força de Ruptura à Tração $(\mathrm{FRT})^{(10)}$, ambos uniaxiais e a introdução de um método biaxial, a Tensão Resultante Final de Ruptura à Tração $(\mathrm{TRF})^{(11)}$. No interior das alças, conteúdos intraluminares exercem tensões sobre a parede intestinal em várias direções que podem ser representadas pelos componentes circunferencial, longitudinal, radial e as suas resultantes. Para o cálculo da TRF foram utilizadas as tensões supracitadas, menos a tensão radial em função do seu valor ínfimo.

A escolha do material polivinilcloreto baseou-se na facilidade de obtenção, no custo e na facilidade de manuseio. Além disso, o P.V.C. praticamente não adere nas estruturas intra-abdominais, sendo dessa forma utilizado nas cirurgias de emergência para o controle do quadro de síndrome compartimental do abdômen pós-trauma e recebe o nome de "Bolsa de Bogotá"(15). A imersão do segmento de alça em solução de cloridrato de papaverina antes dos testes foi utilizada com o intuito de reduzir o grau de espasmo instalado na alça após o manuseio $^{(10)}$. 
Independentemente do teste biomecânico aplicado, os subgrupos com aderências intactas apresentaram maior resistência à ruptura em relação aos subgrupos com aderências removidas. Os subgrupos com ausência de formação de aderências ao redor das anastomoses pela proteção da película de P.V.C. apresentaram a menor resistência mecânica na linha de sutura. Essa diferença marcante na resistência tênsil entre os subgrupos talvez possa ser justificada em função de que as aderências intactas sobre as anastomoses propiciaram influências benéficas biológicas e mecânicas durante os cinco dias de pós-operatório, enquanto que nos subgrupos com aderências removidas, receberam somente os efeitos benéficos biológicos dessas estruturas, pois, com a sua retirada antes dos testes, o efeito da ação mecânica foi provavelmente removido. Além disso, por mais delicada que seja a técnica empregada na retirada das aderências, micro-traumas são gerados sobre a linha de sutura, enfraquecendo-a ${ }^{(14)}$. Por final, os subgrupos com ausência da formação de aderências sobre as anastomoses apresentaram os menores valores de resistência mecânica devido a falta de atuação tanto da ação biológica quanto da ação mecânica das mesmas durante todo o período experimental.

Cabe ressaltar que estudos em áreas de cicatrização intestinal necessitam de especificações mais minuciosas quanto ao tratamento dado às aderências nas linhas de sutura durante os testes de resistência ou a qualquer outro tipo de avaliação. Esse fato é importante, pois as aderências repercutem diretamente no processo de reparação tecidual, agindo como variável e podendo modificar os resultados obtidos pelos testes. Sendo assim, quando o foco do estudo não for a ação das aderências sobre as anastomoses, acreditamos que os resultados seriam mais precisos se não ocorressem a formação das aderências nas linhas de suturas e para esse fim, a película de P.V.C. sobre a anastomose poderia ser útil, tornando mais homogêneos os espécimes sob teste.

As diferenças metodológicas fazem com que os resultados colhidos neste trabalho seja de difícil confrontação com os resultados de outros trabalhos. Mesmo assim, pesquisas empregando os mesmos conceitos físicos-mecânicos chegaram a resultados similares, mostrando que as aderências aumentam a resistência da anastomose, principalmente na fase inicial do processo de cicatrização intestinal ${ }^{(4,5,6,14)}$.

\section{Conclusões}

As análises obtidas dos resultados deste trabalho permitem concluir que:
- As aderências abdominais exerceram influências no aumento da resistência sobre as anastomoses cólicas em rato no quinto dia pós-operatório

- A película de polivinilcloreto pode servir como superfície de separação entre a linha de sutura e as outras estruturas abdominais.

- Os testes biomecânicos, Pressão de Ruptura à Distensão por Líquido, Força de Ruptura à Tração e Tensão Resultante Final de Ruptura à Tração são válidos para o estudo da resistência de ruptura e reparação tecidual em anastomoses cólicas em ratos no quinto dia pós-operatório.

\section{Referências}

1. Medeiros RR. Emprego experimental do bisturi elétrico nas anastomoses do intestino grosso. Estudo da cicatrização à microscopia óptica e de polarização [Tese - Livre-Docência ]. Faculdade de Ciências Médicas da UNICAMP; 1982.

2. Cartier R, Brunette I, Hashimoto K, Bourne WN, Schaff HV. Angiogenic factor: A possible mechanism for neovascularization produced by omental pedicles. J Thorac Cardiov Surg 1990; 99: 264-8.

3. Fagundes J.J. - Estudo comparativo da cicatrização de anastomoses cólicas realizadas com auxílio do bisturi laser de dióxido de carbono: trabalho experimental em cães. Campinas [Tese - Doutorado] Faculdade de Ciências Médicas da UNICAMP; 1990.

4. Adams W, Ctercteko G, Bilous M. Effect of an omental wrap on the healing and vascularity of compromised intestinal anastomoses. Dis Colon Rectum 1992; 35: 731-8.

5. Garcia-Olmo D, Lucas FJ, Paya J. Relationship between peritoneal adhesions phenomena and the experimental resistance of colonic anastomoses: influence of Omentoplasty. Eur Surg Res 1996; 28: 315-22.

6. Aprilli F, Guimarães AS, Rocha JJR. Anastomoses intestinais. In: Silva Jr OC. Modelos experimentais de pesquisa em cirurgia. 1ed. São Paulo: Robe Editorial; 1998.

7. Christen D, Buckmann RF. Peritoneal adhesions after laparotomy: prophylatic mesures. Hepatogastroenterol 1991; 38: 283-6.

8. Jiborn H, Ahonen J, Zederfeldt B. Healing of experimental colonic anastomoses. I. Bursting strength of the colon after left colon resection and anastomosis. Am J Surg 1978; 136: 587-94.

9. Christensen H, Langfelt S, Laurberg S. Bursting strength of experimental colonic anastomoses: a methodological study. Eur Surg Res 1993; 25: 38-45.

10. Naresse, LE. Efeito da peritonite fecal na cicatrização do cólon distal no rato: avaliação anátomo-patológica, estudo da força de ruptura e da hidroxiprolina tecidual [Tese - Doutorado]. Faculdade de Medicina da UNESP; 1990.

11. Wu, FC. Estudo da ação de aderências sobre a anastomose cólica: trabalho experimental em ratos [Dissertação Mestrado]. Faculdade de Ciências Médicas da UNICAMP; 2000.

12. Nash, WA. Flexão e torção composta; teoria das falhas. In: Resistência dos materiais. 2ed. São Paulo: McGraw-Hill; 1982.

13. DeCherney AH, diZerega GS. Clinical problem of intraperitoneal postsurgical adhesion formation following general surgery and the use of adhesion prevention barriers. Surg Clin North Am 1997; 77: 671-89.

14. Hendricks T, Mastboom WJB. Healing of experimental intestinal anastomosis: parameters for repairs. Dis Colon Rectum 1990; 33: 891-901. 
15. Burch JM, Moore EE, Moore FA, Franciose R. The abdominal compartment syndrome. Surg Clin North Am 1996; 76: 833-42.

\section{Agradecimentos}

Agradecemos aos Biólogos do Núcleo de Cirurgia Experimental-Unicamp, Ana Cristina de Moraes e
William Adalberto Silva, além do Prof. Dr. Luis Roberto Magna (Departamento de Genética Médica) pelos serviços prestados na confecção deste trabalho. Agradecemos também ao LABIC - Laboratório de Inteligência Computacional pelo apoio na digitalização de imagens.

Wu FC, Ayrizono MLS, Fagundes JJ, Coy CSR, Góes JRN, Leonardi LS. Biomechanical study to evaluate the influence of abdominal adhesions in colonic anastomoses: experimental study in rats. Acta Cir Bras [serial online] 2003 May-Jun;18(3). Available from URL: http://www.scielo.br/acb.

ABSTRACT - Purpose: To evaluate the influence of abdominal adhesions in the distal colonic anastomoses in rats using biomechanical tests. Methods: Ninety Wistar-CEMIB (UNICAMP) rats were distributed in 3 groups. In group 1 the analysis was performed by bursting strength test, in group 2 by breaking strength test and in group 3 by final resultant tension test. In each group, 10 animals were characterized by the presence of adhesions in the anastomoses during the biomechanical test performance, 10 animals had the anastomoses adhesions removed before the test and 10 animals were free of adhesions in the anastomoses during the test (protection by P.V.C. sheet in the anastomoses area). Results: Evaluations were made on the 5th post-operatory day and independent of the test performed, the results were similar. The mechanical resistence in the subgroups characterized by the presence of adhesions in anastomoses were higher than subgroups with anastomoses adhesions removed. The subgroups free of adhesions in the anastomoses (P.V.C. protection) demonstrated the lowest mechanical resistance in anastomoses breakdown (Group 1$\mathrm{p}=0,0001$, Group 2-p=0,0003 and Group 3-p=0,0001). Conclusion: Analysis of the results allowed to conclude that abdominal adhesions presented benefic influence in the distal colonic wound healing process in rats thus increasing mechanical resistance of the anastomoses.

KEY WORDS - Healing. Colon. Adhesions. Anastomose. Biomechanic.

Conflito de interesse: nenhum Fonte de financiamento: CAPES

Correspondência:

Feng Chung Wu

Rua Lontra, 26

85861-120 Foz do Iguaçu - PR

wufc@ig.com.br

Data do recebimento: 08/02/2003

Data da revisão: 21/02/2003

Data da aprovação: 03/03/2003 